\title{
On definitions of the pluricomplex Green function
}

\author{
by Armen Edigarian (Kraków)
}

\begin{abstract}
We give several definitions of the pluricomplex Green function and show their equivalence.
\end{abstract}

1. Introduction. We denote by $E$ the unit disc in $\mathbb{C}$. Let $D$ be a domain in $\mathbb{C}^{n}$. Put

$g_{D}(a, z):=\sup \{u(z): u \in P S H(D), u<0$,

$\exists M, r>0: u(w) \leq M+\log \|w-a\|, w \in B(a, r) \subset D\}, \quad a, z \in D$,

where $\operatorname{PSH}(D)$ denotes the set of all plurisubharmonic functions on $D$ and $B(a, r)$ denotes the ball with center at $a$ and radius $r$. The function $g_{D}$ has been introduced by M. Klimek (cf. $[\mathrm{K}])$ and is called the pluricomplex Green function.

In this paper we give several equivalent definitions of the pluricomplex Green function.

Following E. Poletsky (cf. [P-S], [P1], [P2]) for a domain $D \subset \mathbb{C}^{n}$ and $a, z \in D, a \neq z$, we define

$$
\begin{aligned}
& g_{D}^{1}(a, z):=\inf \left\{\sum_{\lambda \in \varphi^{-1}(a)} \operatorname{ord}_{\lambda}(\varphi-a) \log |\lambda|:\right. \\
&\varphi \in \mathcal{O}(E, D), a \in \varphi(E), \varphi(0)=z\}, \\
& g_{D}^{2}(a, z):=\inf \left\{\sum_{\lambda \in \varphi^{-1}(a)} \operatorname{ord}_{\lambda}(\varphi-a) \log |\lambda|:\right. \\
&\varphi \in \mathcal{O}(\bar{E}, D), a \in \varphi(E), \varphi(0)=z\},
\end{aligned}
$$

1991 Mathematics Subject Classification: Primary 32H15, 32F05; Secondary 30C25, 30D50.

Key words and phrases: pluricomplex Green function, Blaschke product, Riesz representation.

Research supported by KBN grant No 2 PO3A 06008. 


$$
\begin{aligned}
& g_{D}^{3}(a, z):=\inf \left\{\sum_{\lambda \in \varphi^{-1}(a)} \log |\lambda|: \varphi \in \mathcal{O}(E, D), a \in \varphi(E), \varphi(0)=z\right\}, \\
& g_{D}^{4}(a, z):=\inf \left\{\sum_{\lambda \in \varphi^{-1}(a)} \log |\lambda|: \varphi \in \mathcal{O}(\bar{E}, D), a \in \varphi(E), \varphi(0)=z\right\},
\end{aligned}
$$

where $\mathcal{O}(E, D)$ denotes the set of all holomorphic mappings $E \rightarrow D$ and $\operatorname{ord}_{\lambda}(\varphi-a)$ denotes the order of vanishing of $\varphi-a$ at $\lambda$. Note that in the whole paper for any holomorphic mapping $\varphi: \bar{E} \rightarrow D$ by $\varphi^{-1}(a)$ we mean $\varphi^{-1}(a) \cap E$ and it is always a finite set provided $\varphi$ is nonconstant.

We put $g_{D}^{1}(a, a)=g_{D}^{2}(a, a)=g_{D}^{3}(a, a)=g_{D}^{4}(a, a)=-\infty$.

Remarks. 1. For any $z \in D \backslash\{a\}$ there exists $\varphi \in \mathcal{O}(\bar{E}, D)$ such that $\varphi(0)=z$ and $a \in \varphi(E)$ (cf. [J-P], Remark 3.1.1). So, the above functions are well defined.

2. Note that $g_{D}^{1} \leq g_{D}^{2}, g_{D}^{3} \leq g_{D}^{4}, g_{D}^{1} \leq g_{D}^{3}$, and $g_{D}^{2} \leq g_{D}^{4}$.

Define

$$
\begin{aligned}
& k_{D}(a, z):=\inf \{\log \sigma: \exists \varphi \in \mathcal{O}(\bar{E}, D): \varphi(0)=a, \varphi(\sigma)=z, \sigma>0\}, \\
& g_{D}^{5}(a, z):=\inf \left\{\frac{1}{2 \pi} \int_{0}^{2 \pi} k_{D}\left(a, \varphi\left(e^{i \theta}\right)\right) d \theta:\right. \\
&\varphi \in \mathcal{O}(\bar{E}, D), \varphi(0)=z\}, \quad a, z \in D .
\end{aligned}
$$

Note that $g_{D}^{5}(a, \cdot)$ is the envelope of $k_{D}(a, \cdot)$ in the sense of Poletsky (see Theorem 11).

The main result of the paper is the following

Theorem 1. Let $D$ be a domain in $\mathbb{C}^{n}$. Then

$$
g_{D}=g_{D}^{1}=g_{D}^{2}=g_{D}^{3}=g_{D}^{4}=g_{D}^{5} .
$$

Remarks. The most difficult problem in Theorem 1 is the equality $g_{D}=g_{D}^{2}$. It was proved in [P1]. We present a much simpler and complete proof. The equality $g_{D}=g_{D}^{4}$ was stated in [P2].

2. Definitions and auxiliary results. Let $D$ be a domain in $\mathbb{C}^{n}$ and let $\varphi: \bar{E} \rightarrow D$ be a holomorphic mapping. For a point $a \in D$ we define

$$
\begin{aligned}
u_{(\varphi, a)}(\lambda) & :=\sum_{\zeta \in \varphi^{-1}(a)} \operatorname{ord}_{\zeta}(\varphi-a) \log \left|\frac{\lambda-\zeta}{1-\bar{\zeta} \lambda}\right|, \quad \lambda \in E, \\
H(\varphi, a) & :=u_{(\varphi, a)}(0) .
\end{aligned}
$$

For convenience we put $\sum_{\emptyset}=0$ in the whole paper. For a constant mapping 
$\varphi \equiv a$ we put $u_{(\varphi, a)} \equiv-\infty$. In this notation we have

$$
g_{D}^{2}(a, z)=\inf \{H(\varphi, a): \varphi \in \mathcal{O}(\bar{E}, D), \varphi(0)=z\}, \quad a, z \in D .
$$

For the functional $H$ we have the following

Lemma 2. Let $\varphi: \bar{E} \rightarrow D$ and $h: \bar{E} \rightarrow \bar{E}$ be holomorphic mappings. Then for any $a \in D$ such that $\varphi \not \equiv a$ we have

$$
H(\varphi \circ h, a)=\iint_{E} \log |\zeta| \Delta\left(u_{(\varphi, a)} \circ h(\zeta)\right) .
$$

Proof. Note that if $\varphi(h(0))=a$ then

$$
H(\varphi \circ h, a)=\iint_{E} \log |\zeta| \Delta\left(u_{(\varphi, a)} \circ h(\zeta)\right)=-\infty .
$$

So, we may assume that $\varphi(h(0)) \neq a$. Put

$$
\psi_{j}(\lambda):=\frac{h(\lambda)-\lambda_{j}}{1-\bar{\lambda}_{j} h(\lambda)}, \quad \text { where } \lambda_{j} \in \varphi^{-1}(a) .
$$

Note that $\psi_{j} \in \mathcal{O}(\bar{E})$ and $\psi_{j}(0) \neq 0$. Hence using the Jensen formula (see $[\mathrm{R}]$, Theorem 15.18) we have

$$
\log \left|\psi_{j}(0)\right|=\sum_{m=1}^{N} \log \left|\alpha_{m}\right|+\frac{1}{2 \pi} \int_{0}^{2 \pi} \log \left|\psi_{j}\left(e^{i \theta}\right)\right| d \theta,
$$

where $\alpha_{1}, \ldots, \alpha_{N}$ are the zeros of $\psi_{j}$ with multiplicities. But on the other hand by the Riesz representation we have

$$
\log \left|\psi_{j}(0)\right|=\frac{1}{2 \pi} \int_{0}^{2 \pi} \log \left|\psi_{j}\left(e^{i \theta}\right)\right| d \theta+\iint_{E} \log |\zeta| \Delta\left(\log \left|\psi_{j}(\zeta)\right|\right) .
$$

Hence,

$$
\sum_{m=1}^{N} \log \left|\alpha_{m}\right|=\iint_{E} \log |\zeta| \Delta\left(\log \left|\psi_{j}(\zeta)\right|\right)
$$

From this we derive the desired result.

Lemma 3 (cf. [P1], Lemma 3.2). Let $v$ be a plurisubharmonic function in some neighborhood of $\bar{E}^{2}$ such that $v(0,0) \neq-\infty$ and $v\left(0, e^{i \theta}\right) \neq-\infty$, $\theta \in[0,2 \pi)$. Then

$$
\frac{1}{2 \pi} \int_{0}^{2 \pi}\left(\iint_{E} \log |\zeta| \Delta_{\zeta}\left(v\left(e^{i \alpha} \zeta, \zeta\right)\right)\right) d \alpha \leq \frac{1}{2 \pi} \int_{0}^{2 \pi}\left(\iint_{E} \log |\zeta| \Delta_{\zeta} v\left(\zeta, e^{i \theta}\right)\right) d \theta .
$$

Therefore, there exists $\alpha_{0} \in[0,2 \pi)$ such that

$$
\iint_{E} \log |\zeta| \Delta_{\zeta}\left(v\left(e^{i \alpha_{0}} \zeta, \zeta\right)\right) \leq \frac{1}{2 \pi} \int_{0}^{2 \pi}\left(\iint_{E} \log |\zeta| \Delta_{\zeta} v\left(\zeta, e^{i \theta}\right)\right) d \theta .
$$


Proof. By the Riesz representation we have

$$
\begin{aligned}
v(0,0)= & \frac{1}{2 \pi} \int_{0}^{2 \pi} v\left(0, e^{i \theta}\right) d \theta+\iint_{E} \log |\zeta| \Delta_{\zeta} v(0, \zeta) \\
= & \frac{1}{4 \pi^{2}} \int_{0}^{2 \pi} \int_{0}^{2 \pi} v\left(e^{i \alpha}, e^{i \theta}\right) d \alpha d \theta \\
& +\frac{1}{2 \pi} \int_{0}^{2 \pi} d \theta \iint_{E} \log |\zeta| \Delta_{\zeta} v\left(\zeta, e^{i \theta}\right)+\iint_{E} \log |\zeta| \Delta_{\zeta} v(0, \zeta) .
\end{aligned}
$$

Again by the Riesz representation for any fixed $\alpha \in[0,2 \pi)$ we have

$$
v(0,0)=\frac{1}{2 \pi} \int_{0}^{2 \pi} v\left(e^{i(\alpha+\theta)}, e^{i \theta}\right) d \theta+\iint_{E} \log |\zeta| \Delta_{\zeta} v\left(e^{i \alpha} \zeta, \zeta\right) .
$$

Hence, integrating (1) in $\alpha \in[0,2 \pi)$ we obtain

$$
v(0,0)=\frac{1}{4 \pi^{2}} \int_{0}^{2 \pi} \int_{0}^{2 \pi} v\left(e^{i(\alpha+\theta)}, e^{i \theta}\right) d \theta d \alpha+\frac{1}{2 \pi} \int_{0}^{2 \pi}\left[\iint_{E} \log |\zeta| \Delta_{\zeta} v\left(e^{i \alpha} \zeta, \zeta\right)\right] d \alpha .
$$

So,

$$
\begin{aligned}
\frac{1}{2 \pi} \int_{0}^{2 \pi}\left[\iint_{E} \log |\zeta| \Delta_{\zeta} v\left(e^{i \alpha} \zeta, \zeta\right)\right] d \alpha= & \frac{1}{2 \pi} \int_{0}^{2 \pi} d \theta \iint_{E} \log |\zeta| \Delta_{\zeta} v\left(\zeta, e^{i \theta}\right) \\
& +\iint_{E} \log |\zeta| \Delta_{\zeta} v(0, \zeta) \\
\leq & \frac{1}{2 \pi} \int_{0}^{2 \pi} d \theta \iint_{E} \log |\zeta| \Delta_{\zeta} v\left(\zeta, e^{i \theta}\right) .
\end{aligned}
$$

As a corollary we have the following

Lemma 4. Let $\varphi: \bar{E} \rightarrow D$ and $h: \bar{E}^{2} \rightarrow \bar{E}$ be holomorphic mappings. Then for any $a \in D$ such that $a \notin \varphi(h(\{0\} \times \partial E))$ and $\varphi(h(0,0)) \neq a$ there exists $\alpha_{0} \in[0,2 \pi)$ with

$$
H\left(\varphi \circ h\left(e^{i \alpha_{0}} \zeta, \zeta\right), a\right) \leq \frac{1}{2 \pi} \int_{0}^{2 \pi} H\left(\varphi \circ h\left(\zeta, e^{i \theta}\right), a\right) d \theta .
$$

Proof. Take $v:=u_{(\varphi, a)} \circ h$. Then the result follows from Lemmas 2 and 3.

Recall that a holomorphic function $\phi: E \rightarrow E$ is called inner if $\left|\phi^{*}(\zeta)\right|=$ $\lim _{r \rightarrow 1}|\phi(r \zeta)|=1$ for almost all $\zeta \in \partial E$. Any Blaschke product is an inner function. A simple example of an inner function but not a Blaschke product 
is the function $e(\lambda, c):=e^{c(\lambda-1) /(\lambda+1)}, c>0$. It plays an important role in our considerations. Put

$$
l_{k}(\lambda, c)=\frac{\lambda+e^{-c / k}}{1+e^{-c / k} \lambda}, \quad \lambda \in E, c>0, k \in \mathbb{N} .
$$

We have

Lemma 5. (a) For fixed $c>0$ and $\tau \in E \backslash\{0\}$ the function

$$
\phi(\lambda)=\frac{e(\lambda, c)-\tau}{1-\bar{\tau} e(\lambda, c)}
$$

is a Blaschke product.

(b) For fixed $c>0$ we have $l_{k}(\lambda, c) \rightarrow 1$ and $l_{k}^{k}(\lambda, c) \rightarrow e(\lambda, c)$ locally uniformly on $E$ as $k \rightarrow \infty$.

P r o of. (a) Note that $\phi$ is an inner function. By Theorem 2 in Chapter III of $[\mathrm{N}]$, any inner function which has no zero radial limits is a Blaschke product. By simple calculations we see that $\phi$ has no zero radial limits.

(b) It is enough to note that

$$
l_{k}(\lambda, c)=1+\left(1-e^{-c / k}\right) \frac{\lambda-1}{1+e^{-c / k} \lambda} .
$$

Recall the following approximation result:

Lemma 6. Let $F \in \mathcal{C}(V \times \partial E)$ and $F(\cdot, \zeta) \in \mathcal{O}(V), \zeta \in \partial E$, where $V$ is a domain in $\mathbb{C}^{m}$. For $\nu=1,2, \ldots$ put

$$
F_{\nu}(\xi, \zeta):=\frac{1}{2 \pi \nu} \sum_{j=0}^{\nu-1} \sum_{k=-j}^{j}\left(\int_{0}^{2 \pi} \frac{F\left(\xi, e^{i \theta}\right)}{e^{i \theta(k+1)}} d \theta\right) \zeta^{k} .
$$

Then:

(1) $F_{\nu}$ are holomorphic w.r.t. $\xi \in V$ and rational w.r.t. $\zeta$ with pole of order $\leq \nu-1$ at $\zeta=0$;

(2) $\left\{F_{\nu}\right\}$ converges locally uniformly to $F$ on $V \times \partial E$;

(3) if $F(0, \zeta) \equiv 0$, then $F_{\nu}(0, \zeta) \equiv 0, \zeta \in \partial E$.

Proof. It is enough to prove (2), because (1) and (3) are evident.

Put

$$
K_{\nu}(x):=\frac{1}{\nu}\left[\frac{\sin \frac{\nu}{2} x}{\sin \frac{1}{2} x}\right]^{2} .
$$

Then (see [H], Chapter II) $\frac{1}{2 \pi} \int_{0}^{2 \pi} K_{\nu}(\theta) d \theta=1$ and

$$
F_{\nu}\left(\xi, e^{i t}\right)=\frac{1}{2 \pi} \int_{0}^{2 \pi} F\left(\xi, e^{i \theta}\right) K_{\nu}(t-\theta) d \theta=\frac{1}{2 \pi} \int_{0}^{2 \pi} F\left(\xi, e^{i(t-\theta)}\right) K_{\nu}(\theta) d \theta .
$$


For $\delta>0$ we have

$$
\begin{aligned}
F_{\nu}\left(\xi, e^{i t}\right)-F\left(\xi, e^{i t}\right)= & \frac{1}{2 \pi} \int_{-\pi}^{\pi}\left(F\left(\xi, e^{i(t-\theta)}\right)-F\left(\xi, e^{i t}\right)\right) K_{\nu}(\theta) d \theta \\
= & \frac{1}{2 \pi} \int_{-\delta}^{\delta}\left(F\left(\xi, e^{i(t-\theta)}\right)-F\left(\xi, e^{i t}\right)\right) K_{\nu}(\theta) d \theta \\
& +\frac{1}{2 \pi} \int_{\pi>|\theta| \geq \delta}\left(F\left(\xi, e^{i(t-\theta)}\right)-F\left(\xi, e^{i t}\right)\right) K_{\nu}(\theta) d \theta .
\end{aligned}
$$

Suppose that $K=L \times \partial E$, where $L \Subset V$. Then

$$
\begin{aligned}
\mid F_{\nu}\left(\xi, e^{i t}\right) & -F\left(\xi, e^{i t}\right) \mid \\
& \leq \sup _{-\delta<\theta<\delta}\left|F\left(\xi, e^{i(t-\theta)}\right)-F\left(\xi, e^{i t}\right)\right|+2\|F\|_{K} \sup _{\pi>|\theta| \geq \delta} K_{\nu}(\theta),
\end{aligned}
$$

where $\|F\|_{K}:=\sup _{(\xi, \zeta) \in K}|F(\xi, \zeta)|$. Recall that $\lim _{\nu \rightarrow \infty} \sup _{\pi>|\theta| \geq \delta} K_{\nu}(\theta)$ $=0$. Since $F$ is a continuous mapping, we conclude the proof.

3. Proof of Theorem 1. We will prove Theorem 1 in several lemmas. We prove consecutively that $g_{D}^{1}=g_{D}^{2}=g_{D}^{3}=g_{D}^{4}($ Lemma 7$), g_{D}^{5} \geq g_{D}^{2}$ (Lemma 9), $g_{D}=g_{D}^{5}$ (Lemma 10), and finally, $g_{D} \leq g_{D}^{4}$ (Lemma 12). In this way we will have proved Theorem 1 . In the whole section we assume that the domain $D$ and points $a, z \in D$ are fixed. Note that if $a=z$ then the assertion of Theorem 1 is evident, because all the functions are equal to $-\infty$. So, we may assume that $a \neq z$.

Lemma 7. $g_{D}^{1}(a, z)=g_{D}^{2}(a, z)=g_{D}^{3}(a, z)=g_{D}^{4}(a, z)$.

Proof. It is enough to prove that

(1) $g_{D}^{1}(a, z)=g_{D}^{2}(a, z)$,

(2) $g_{D}^{3}(a, z)=g_{D}^{4}(a, z)$,

(3) $g_{D}^{2}(a, z)=g_{D}^{4}(a, z)$.

(1)-(2) We know that $g_{D}^{1}(a, z) \leq g_{D}^{2}(a, z)\left(\right.$ resp. $\left.g_{D}^{3}(a, z) \leq g_{D}^{4}(a, z)\right)$. Fix $A>g_{D}^{1}(a, z)$ (resp. $\left.A>g_{D}^{3}(a, z)\right)$.

There exists a holomorphic mapping $\varphi: E \rightarrow D$ such that $\varphi(0)=z$, $a \in \varphi(E)$, and

$$
\sum_{\lambda \in \varphi^{-1}(a)} \operatorname{ord}_{\lambda}(\varphi-a) \log |\lambda|<A \quad\left(\text { resp. } \sum_{\lambda \in \varphi^{-1}(a)} \log |\lambda|<A\right) .
$$

Let $\varphi^{-1}(a)=\left\{\lambda_{j}: j=1,2, \ldots\right\}$, where $\lambda_{j}$ 's are counted with multiplicities (resp. without multiplicities). We may assume that $\left|\lambda_{1}\right| \leq\left|\lambda_{2}\right| \leq \ldots$ There exists $N>0$ such that $\sum_{j=1}^{N} \log \left|\lambda_{j}\right|<A$. Let $\widetilde{\varphi}(\lambda)=\varphi(R \lambda)$, where 
$R \in\left(\left|\lambda_{N}\right|, 1\right)$. Note that $\widetilde{\varphi} \in \mathcal{O}(\bar{E}, D)$ and $\widetilde{\varphi}(0)=z$. Then we have

$$
\begin{gathered}
\sum_{\lambda \in \widetilde{\varphi}^{-1}(a)} \operatorname{ord}_{\lambda}(\widetilde{\varphi}-a) \log |\lambda| \leq \sum_{j=1}^{N}\left(\log \left|\lambda_{j}\right|-\log R\right) \\
\left.\quad \text { resp. } \sum_{\lambda \in \widetilde{\varphi}^{-1}(a)} \log |\lambda| \leq \sum_{j=1}^{N}\left(\log \left|\lambda_{j}\right|-\log R\right)\right) .
\end{gathered}
$$

So, if $R$ is close enough to 1 then

$$
\begin{gathered}
g_{D}^{2}(a, z) \leq \sum_{\lambda \in \widetilde{\varphi}^{-1}(a)} \operatorname{ord}_{\lambda}(\widetilde{\varphi}-a) \log |\lambda|<A \\
\text { (resp. } \left.g_{D}^{4}(a, z) \leq \sum_{\lambda \in \widetilde{\varphi}^{-1}(a)} \log |\lambda|<A\right) .
\end{gathered}
$$

Hence, $g_{D}^{2}(a, z) \leq g_{D}^{1}(a, z)\left(\right.$ resp. $\left.g_{D}^{4}(a, z) \leq g_{D}^{3}(a, z)\right)$.

(3) Let $\varphi: \bar{E} \rightarrow D$ be a holomorphic mapping such that $\varphi(0)=z \neq a$ and $a \in \varphi(E)$. Suppose that $\varphi(\mu)=a$ and $\operatorname{ord}_{\mu}(\varphi-a)=m$. Note that $\mu \neq 0$. Let

$$
\psi(\lambda):=\frac{\varphi(\lambda)-a}{(\lambda-\mu)^{m}}\left(\lambda-\mu_{1}\right) \ldots\left(\lambda-\mu_{m}\right)+a, \quad \lambda \in E,
$$

where $\mu_{1}, \ldots, \mu_{m}$ are pairwise different, $\mu_{1} \ldots \mu_{m}=\mu^{m}$, and $\mu_{1}, \ldots, \mu_{m}$ are very close to $\mu\left({ }^{1}\right)$. Note that if $\mu_{1}, \ldots, \mu_{m}$ are close enough to $\mu$ then $\psi \in \mathcal{O}(\bar{E}, D)$ and $\psi(0)=\varphi(0)=z$. Moreover, $\psi\left(\lambda_{0}\right)=a$ iff $\varphi\left(\lambda_{0}\right)=a$ and $\lambda_{0} \neq \mu$, or $\lambda_{0} \in\left\{\mu_{1}, \ldots, \mu_{m}\right\}$, and

$$
\sum_{\lambda \in \varphi^{-1}(a)} \operatorname{ord}_{\lambda}(\varphi-a) \log |\lambda|=\sum_{\substack{\lambda \in \psi^{-1}(a) \\ \lambda \notin\left\{\mu_{1}, \ldots, \mu_{m}\right\}}} \operatorname{ord}_{\lambda}(\psi-a) \log |\lambda|+\sum_{j=1}^{m} \log \left|\mu_{j}\right| .
$$

Note that the multiplicities of $\psi$ at $\mu_{j}, j=1, \ldots, m$, are equal to 1 . Applying this technique $N$ times, where $N$ is the number of zeros of $\varphi-a$ in $E$, we obtain the result.

The following result is basic for the proof of Theorem 1 .

LeMma 8. Let $\Phi: \bar{E} \rightarrow D$ be a holomorphic mapping such that $\Phi(0)=z$ and $a \notin \Phi(\partial E)$. Then

$$
\frac{1}{2 \pi} \int_{0}^{2 \pi} k_{D}\left(a, \Phi\left(e^{i \theta}\right)\right) d \theta \geq g_{D}^{2}(a, z)
$$

$\left({ }^{1}\right)$ For instance, if $\mu=r e^{i \theta}$ then let $\mu_{j}=r e^{i \theta_{j}}, j=1, \ldots, m$, where $\theta_{1}, \ldots, \theta_{m}$ are pairwise different, close to $\theta$, and such that $\theta_{1}+\ldots+\theta_{m}=m \theta$. 
R e m a r k. From the definitions we see that $k_{D}(a, w) \geq g_{D}^{2}(a, w), w \in D$. So, a priori (2) states less than the subaverage property of the function $g_{D}^{2}(a, \cdot)$. But it turns out that $(2)$ is sufficient to show that $g_{D}^{2}(a, \cdot)$ is a plurisubharmonic function, hence has the subaverage property. It is worth noting that we assume that $-\infty$ is a plurisubharmonic function.

Before we present the proof of Lemma 8 note the following immediate corollary.

Lemma 9. $g_{D}^{5}(a, z) \geq g_{D}^{2}(a, z)$.

Proof of Lemma 8 . Take any $A \in \mathbb{R}$ such that

$$
\frac{1}{2 \pi} \int_{0}^{2 \pi} k_{D}\left(a, \Phi\left(e^{i \theta}\right)\right) d \theta<A .
$$

It is sufficient to show that $g_{D}^{2}(a, z) \leq A$. Note that $k_{D}(a, \Phi(\cdot))$ is an upper semicontinuous function in $\bar{E}$ (see the proof of Lemma 10). Hence, we can find a continuous function $q: \partial E \rightarrow \mathbb{R}$ such that $k_{D}(a, \Phi(\xi))<q(\xi), \xi \in \partial E$, and

$$
\frac{1}{2 \pi} \int_{0}^{2 \pi} q\left(e^{i \theta}\right) d \theta<A .
$$

For any $\xi \in \partial E$ there exist $\varphi_{\xi} \in \mathcal{O}(\bar{E}, D)$ and $\sigma_{\xi} \in(0,1)$ such that $\varphi_{\xi}(0)=$ $\Phi(\xi), \varphi_{\xi}\left(\sigma_{\xi}\right)=a$, and

$$
\log \sigma_{\xi}<q(\xi) .
$$

Note that for any $\xi \in \partial E$ there exists $t(\xi)>0$ such that for any $\zeta \in$ $B(\xi, t(\xi))$ we may define a mapping $\varphi_{\xi, \zeta} \in \mathcal{O}(\bar{E}, D)$ as follows:

$$
\varphi_{\xi, \zeta}(\lambda):=\varphi_{\xi}(\lambda)+(\Phi(\zeta)-\Phi(\xi))\left(1-\lambda / \sigma_{\xi}\right), \quad \lambda \in \bar{E} .
$$

Observe that $\varphi_{\xi, \zeta}(0)=\Phi(\zeta)$ and $\varphi_{\xi, \zeta}\left(\sigma_{\xi}\right)=\varphi_{\xi}\left(\sigma_{\xi}\right)=a$. Taking smaller $t(\xi)>0$ if necessary we have

$$
\log \sigma_{\xi}<q(\zeta), \quad \zeta \in B(\xi, t(\xi)),
$$

and $\varphi_{\xi, \zeta}(\bar{E}) \Subset D$ for any $\zeta \in \partial E \cap B(\xi, t(\xi))$. Taking even smaller $t(\xi)$, we may choose $\xi_{1}, \ldots, \xi_{l}$ such that $\partial E \subset V_{\xi_{1}} \cup \ldots \cup V_{\xi_{l}}$ and $V_{\xi_{k}} \cap V_{\xi_{j}}=\emptyset$ if $1<|k-j|<l-1, k, j=1, \ldots, l$, where $V_{\xi_{j}}:=B(\xi, t(\xi))$. We put $\delta:=\min _{j=1, \ldots, l} \sigma_{\xi_{j}}$ and $C:=\|q\|$.

Fix $\varepsilon>0$. Note that there exists $r_{1}>1$ such that $\Phi, \varphi_{\xi_{j}, \zeta} \in \mathcal{O}\left(r_{1} E, D\right)$ for $\zeta \in V_{\xi_{j}}, j=1, \ldots, l$. We may assume that $\log r_{1}<\varepsilon$. Take $0<t^{\prime}\left(\xi_{j}\right)<$ $t\left(\xi_{j}\right), j=1, \ldots, l$, such that for $I_{j}:=\partial E \cap \overline{B\left(\xi_{j}, t^{\prime}\left(\xi_{j}\right)\right)}$ we have $I_{j} \cap I_{k}=\emptyset$ for $j \neq k$ and $m\left(\bigcup_{j=1}^{l} I_{j}\right)>2 \pi-\varepsilon$, where $m$ denotes the Lebesgue measure on $\partial E$. Take a closed subset $\Gamma \subset \bigcup I_{j}$ and a continuous function $\tau: \partial E \rightarrow[0,1]$ such that $m(\Gamma)>2 \pi-\varepsilon, \tau=1$ on $\Gamma$, and $\tau=0$ outside $\bigcup I_{j}$. 
For $\zeta \in \partial E$ put

$$
\sigma(\zeta):= \begin{cases}\sigma_{\xi_{j}} / \tau(\zeta) & \text { if } \sigma_{\xi_{j}} / r_{1}<\tau(\zeta) \text { and } \zeta \in I_{j} \\ r_{1} & \text { otherwise. }\end{cases}
$$

Note that $\sigma$ is a continuous function on $\partial E$ and if $\sigma(\zeta)<r_{1}$ then $\tau(\zeta) \sigma(\zeta)=\sigma_{\xi_{j}}$.

For $\lambda \in r_{1} E$ and $\zeta \in \partial E$ we put

$$
\psi(\lambda, \zeta):= \begin{cases}\varphi_{\xi_{j}, \zeta}(\tau(\zeta) \lambda) & \text { if } \zeta \in I_{j}, \\ \Phi(\zeta) & \text { if } \zeta \notin \bigcup_{j=1}^{l} I_{j} .\end{cases}
$$

Note that $\psi(\lambda, \zeta)$ is holomorphic with respect to $\lambda$ and continuous with respect to $(\lambda, \zeta)$. Moreover, $\psi(\cdot, \zeta) \in \mathcal{O}\left(r_{1} E, D\right)$ and $\psi(0, \zeta)=\Phi(\zeta)$ when $\zeta \in \partial E$,

$$
\psi(\sigma(\zeta), \zeta)=a \quad \text { if } \sigma(\zeta)<r_{1}
$$

and

$$
\begin{aligned}
\frac{1}{2 \pi} \int_{0}^{2 \pi} \log \sigma\left(e^{i \theta}\right) d \theta & <\frac{1}{2 \pi} \int_{\Gamma} \log \sigma\left(e^{i \theta}\right) d \theta+\log r_{1} \\
& <\frac{1}{2 \pi} \int_{0}^{2 \pi} q\left(e^{i \theta}\right) d \theta+\varepsilon-\frac{1}{2 \pi} \int_{[0,2 \pi) \backslash \Gamma} q\left(e^{i \theta}\right) d \theta \\
& <A+\varepsilon+C \varepsilon .
\end{aligned}
$$

Now we want to approximate $\psi$ and $\sigma$ by holomorphic (actually meromorphic) mappings. But applying Lemma 6 to $\psi$ and $\sigma$ we may loose the important relation (3). So, we "separate" in $\psi$ the part related to (3). Namely, we have

$$
\psi(\lambda, \zeta)=a \frac{\lambda}{\sigma(\zeta)}+\left(1-\frac{\lambda}{\sigma(\zeta)}\right) \Phi(\zeta)+(\lambda-\sigma(\zeta)) \psi_{0}(\lambda, \zeta),
$$

where

$$
\psi_{0}(\lambda, \zeta):=\frac{\psi(\lambda, \zeta)-a \frac{\lambda}{\sigma(\zeta)}}{\lambda-\sigma(\zeta)}+\frac{\Phi(\zeta)}{\sigma(\zeta)}
$$

Note that $\psi_{0}(\lambda, \zeta)$ extends as a continuous mapping in $r_{1} E \times \partial E$ and holomorphic with respect to $\lambda$.

We denote by $\sigma_{\nu}(\zeta)$ and $\psi_{0 \nu}(\lambda, \zeta)$ the approximations of $\sigma(\zeta)$ and $\psi_{0}(\lambda, \zeta)$ given by Lemma 6 and define

$$
\psi_{\nu}(\lambda, \zeta):=a \frac{\lambda}{\sigma_{\nu}(\zeta)}+\left(1-\frac{\lambda}{\sigma_{\nu}(\zeta)}\right) \Phi(\zeta)+\left(\lambda-\sigma_{\nu}(\zeta)\right) \psi_{0 \nu}(\lambda, \zeta) .
$$


If $\nu$ is large enough, then

- $\min _{\zeta \in \partial E}\left|\sigma_{\nu}(\zeta)\right|>\delta / 2$,

- $\psi_{\nu}(\cdot, \zeta) \in \mathcal{O}\left(r_{2} E, D\right)$ for $\zeta \in \partial E$, where $1<r_{2}<r_{1}$,

- $\max _{\zeta \in \Gamma}\left|\sigma_{\nu}(\zeta)\right|<1$,

- $\frac{1}{2 \pi} \int_{0}^{2 \pi} \log \left|\sigma_{\nu}\left(e^{i \theta}\right)\right| d \theta<\frac{1}{2 \pi} \int_{0}^{2 \pi} \log \sigma\left(e^{i \theta}\right) d \theta+\varepsilon<A+2 \varepsilon+C \varepsilon$.

We fix $\nu$ so large that the above conditions are satisfied.

Note that there exists $\varrho>1$ such that $\min _{1 / \varrho<|\zeta|<\varrho}\left|\sigma_{\nu}(\zeta)\right|>\delta / 2$, and, therefore $\psi_{\nu}\left(\sigma_{\nu}(\zeta), \zeta\right)=a$ if $1 / \varrho<|\zeta|<\varrho$.

Let $\zeta_{1}, \zeta_{2}, \ldots$ be the zeros of $\sigma_{\nu}$ in $E$ counted with multiplicity. Note that $\left|\zeta_{j}\right|<1 / \varrho$ and it is a finite sequence. It is easy to see from Lemma 6 that

$$
\zeta^{2 \nu-2} \prod\left(\frac{\zeta-\zeta_{j}}{1-\bar{\zeta}_{j} \zeta}\right) \psi_{\nu}(\lambda, \zeta)
$$

is a holomorphic mapping in $\left(r_{3} E\right)^{2}$, where $1<r_{3}<\min \left\{r_{2}, \varrho\right\}$. We know that $\psi_{\nu}(0, \zeta)=\Phi(\zeta)$ and, therefore, $\psi_{\nu}(0, \cdot)$ is a holomorphic mapping on $r_{3} E$. Hence, for any $k \geq 2 \nu-2$,

$$
f(\lambda, \zeta):=\psi_{\nu}\left(\lambda \zeta^{k} \prod\left(\frac{\zeta-\zeta_{j}}{1-\bar{\zeta}_{j} \zeta}\right), \zeta\right)
$$

is a holomorphic mapping in $\left(r_{4} E\right)^{2}$, where $1<r_{4}<r_{3}$ is such that

$$
\lambda \zeta^{k} \prod\left(\frac{\zeta-\zeta_{j}}{1-\bar{\zeta}_{j} \zeta}\right) \in r_{3} E \quad \text { for }(\lambda, \zeta) \in\left(r_{4} E\right)^{2} .
$$

Note that $r_{4}$ depends on $k$. We want to show that we can take $k$ so large that $f \in \mathcal{O}\left(\left(r_{4} E\right)^{2}, D\right)$. Note that there exists a neighborhood $W_{1} \subset \mathbb{C}$ of $\partial E$ such that $\psi_{\nu}\left(r_{3} E \times W_{1}\right) \subset D$ and a neighborhood $W_{2} \subset \mathbb{C}$ of 0 such that $\psi_{\nu}\left(W_{2} \times r_{3} E\right) \subset D$. We can take $k$ so large that

$$
\left(\lambda \zeta^{k} \prod\left(\frac{\zeta-\zeta_{j}}{1-\bar{\zeta}_{j} \zeta}\right), \zeta\right) \in\left(r_{3} E \times W_{1}\right) \cup\left(W_{2} \times r_{3} E\right) \quad \text { if }(\lambda, \zeta) \in\left(r_{4} E\right)^{2} .
$$

For such fixed $k$ we have $f \in \mathcal{O}\left(\left(r_{4} E\right)^{2}, D\right)$. Put

$$
\widetilde{\sigma}(\zeta):=\frac{\sigma_{\nu}(\zeta)}{\zeta^{k} \prod\left(\frac{\zeta-\zeta_{j}}{1-\bar{\zeta}_{j} \zeta}\right)} .
$$

Let us collect the facts that we have just proved and that we shall need in the sequel (we change the notation, putting $\sigma$ in place of $\widetilde{\sigma}$ and $r_{0}$ in place of $r_{4}$ ).

There exist a holomorphic mapping $f:\left(r_{0} E\right)^{2} \rightarrow D, r_{0}>1$, and a holomorphic function $\sigma \in \mathcal{O}\left(r_{0} E \backslash\left(1 / r_{0}\right) \bar{E}\right)$ such that 
- $\frac{1}{2 \pi} \int_{0}^{2 \pi} \log \left|\sigma\left(e^{i \theta}\right)\right| d \theta<A+2 \varepsilon+C \varepsilon$,

- $f(\sigma(\zeta), \zeta)=a$ if $|\sigma(\zeta)|<r_{0}$ and $1 / r_{0}<|\zeta|<r_{0}$

- $\min _{\zeta \in \partial E}|\sigma(\zeta)|>\delta / 2$,

- $\max _{\zeta \in \Gamma}|\sigma(\zeta)|<1$,

- $f(0, \zeta)=\Phi(\zeta), \zeta \in r_{0} E$.

Note that $f(0,0)=\Phi(0)=z$ and $a \notin f(\{0\} \times \partial E)$, hence there exists $\varrho_{0}>0$ such that $a \neq f(\xi, \zeta)$ for any $\xi \in \varrho_{0} E$ and any $\zeta \in \mathbb{C}$ such that $1-\varrho_{0}<|\zeta|<1+\varrho_{0}$.

Fix $\zeta_{0} \in \Gamma$ and $\eta_{0} \in \partial E$. For $c>0$ consider the function

$$
\phi_{c}(\lambda):=\frac{\eta_{0} e(\lambda, c)-\sigma\left(\zeta_{0}\right)}{1-\bar{\sigma}\left(\zeta_{0}\right) \eta_{0} e(\lambda, c)} .
$$

We have $\left|\sigma\left(\zeta_{0}\right)\right|<1$, so $\phi_{c}$ is holomorphic in $E$. But also $\sigma\left(\zeta_{0}\right) \neq 0$, hence by Lemma $5, \phi_{c}$ is a Blaschke product. Therefore $\left|\phi_{c}(0)\right|=\prod_{j=1}^{\infty}\left|\lambda_{j}\right|$, where the $\lambda_{j}$ are the zeros of $\phi_{c}$ counted with multiplicity. Note that

$$
\left|\phi_{c}(0)\right|=\left|\frac{\eta_{0} e^{-c}-\sigma\left(\zeta_{0}\right)}{1-\bar{\sigma}\left(\zeta_{0}\right) \eta_{0} e^{-c}}\right| \rightarrow\left|\sigma\left(\zeta_{0}\right)\right| \quad \text { as } c \rightarrow \infty .
$$

So, there exists $c>0$ such that $\log \left|\phi_{c}(0)\right|<\log \left|\sigma\left(\zeta_{0}\right)\right|+\varepsilon$ and $e^{-c}<\varrho_{0}$. Fix such a $c>0$. We can take $s \in \mathbb{N}$ so large that

$$
\sum_{j=1}^{s} \log \left|\lambda_{j}\right|<\log \left|\sigma\left(\zeta_{0}\right)\right|+\varepsilon .
$$

We may find $r<1$ such that

$$
\sum_{j=1}^{s} \log \frac{\left|\lambda_{j}\right|}{r}<\log \left|\sigma\left(\zeta_{0}\right)\right|+\varepsilon,
$$

and $\max _{j=1, \ldots, s}\left|\lambda_{j}\right|<r<1$. Fix such an $r<1$.

There is a neighborhood $U_{0}$ of $\zeta_{0}$ such that $|\sigma(\zeta)|<1$ for $\zeta \in U_{0}$. By Lemma 5 for large enough $k$ we have $\zeta_{0} l_{k}(r \xi, c) \in U_{0}$. Therefore, for $\xi \in \partial E$ we have

$$
f\left(\sigma\left(\zeta_{0} l_{k}(r \xi, c)\right), \zeta_{0} l_{k}(r \xi, c)\right)=a .
$$

Consider the functions $g_{k}(\xi)=\eta_{0} l_{k}^{k}(r \xi, c)-\sigma\left(\zeta_{0} l_{k}(r \xi, c)\right)$ and $g_{\infty}(\xi)=$ $\eta_{0} e(r \xi, c)-\sigma\left(\zeta_{0}\right)$ for $\xi \in \bar{E}$. Note that $g_{k} \rightarrow g_{\infty}$ uniformly on $E$. We know that $g_{\infty}\left(\lambda_{j} / r\right)=0, j=1, \ldots, s$. By the Hurwitz theorem for large enough $k$ we know that $g_{k}$ has zeros $\lambda_{1}^{\prime} / r, \ldots, \lambda_{s}^{\prime} / r$ close to $\lambda_{1} / r, \ldots, \lambda_{s} / r$ such that

$$
\sum_{j=1}^{s} \log \frac{\left|\lambda_{j}^{\prime}\right|}{r}<\log \left|\sigma\left(\zeta_{0}\right)\right|+\varepsilon
$$


So, $f\left(\eta_{0} l_{k}^{k}\left(\lambda_{j}^{\prime}, c\right), \zeta_{0} l_{k}\left(\lambda_{j}^{\prime}, c\right)\right)=a, j=1, \ldots, s$ (use (4)). Therefore, for large enough $k$ it follows that $1-\varrho_{0}<e^{-c / k}$ and

$$
H\left(f\left(\eta_{0} l_{k}^{k}(r \xi, c), \zeta_{0} l_{k}(r \xi, c)\right), a\right)<\log \left|\sigma\left(\zeta_{0}\right)\right|+\varepsilon .
$$

Hence, for any fixed $\zeta_{0} \in \Gamma$ and $\eta_{0} \in \partial E$ there exist $k \in \mathbb{N}$ and $r<1, c>0$ such that (5) is satisfied. Therefore we may find $k \in \mathbb{N}, r<1, c>0$, and $Q \subset \partial E \times \Gamma$ such that $m(Q)>4 \pi^{2}-4 \pi \varepsilon$ and for any $(\eta, \zeta) \in Q,(5)$ is satisfied, $e^{-c}<\varrho_{0}$, and $1-\varrho_{0}<e^{-c / k}$.

Let $Q^{*}$ denote the image of $Q$ under the mapping $(\eta, \zeta) \rightarrow\left(\eta \zeta^{-k}, \zeta\right)$. The Jacobian of this mapping is equal to 1 on $\partial E \times \partial E$, hence $m\left(Q^{*}\right)=m(Q)$. So, there exists $\nu \in \partial E$ such that

$$
m\left(\left\{\zeta \in \partial E:(\nu, \zeta) \in Q^{*}\right\}\right)>2 \pi-2 \varepsilon .
$$

Note that

$$
H\left(f\left(\nu \zeta^{k} l_{k}^{k}(r \xi, c), \zeta l_{k}(r \xi, c)\right), a\right)<\log |\sigma(\zeta)|+\varepsilon
$$

on $S:=\left\{\zeta \in \partial E:(\nu, \zeta) \in Q^{*}\right\} \subset \Gamma$ and $m(S)>2 \pi-2 \varepsilon$. Consider the mapping $\varphi(\xi):=f\left(\nu \xi^{k}, \xi\right), \xi \in \bar{E}$. Note that $\varphi(0)=f(0,0)=\Phi(0)=z$. Put

$$
h(\xi, \zeta)=\zeta l_{k}(r \xi, c)=\zeta \frac{r \xi+e^{-c / k}}{1+r e^{-c / k} \xi}, \quad \xi, \zeta \in \partial E .
$$

Note that $h(\xi, \zeta) \in \mathcal{O}\left(\bar{E}^{2}\right), a \notin \varphi(h(\{0\} \times \partial E))$, and $\varphi(h(0,0))=z \neq a$. Therefore, by Lemma 4 there exists $\alpha_{0} \in[0,2 \pi)$ such that

$$
H\left(\varphi \circ h\left(e^{i \alpha_{0}} \zeta, \zeta\right), a\right) \leq \frac{1}{2 \pi} \int_{0}^{2 \pi} H\left(\varphi \circ h\left(\zeta, e^{i \theta}\right), a\right) d \theta .
$$

Put $\widetilde{\varphi}(\xi):=\varphi\left(h\left(e^{i \alpha_{0}} \xi, \xi\right)\right)$. Then $\widetilde{\varphi} \in \mathcal{O}(\bar{E}, D), \widetilde{\varphi}(0)=z$, and

$$
\begin{aligned}
H(\widetilde{\varphi}, a) & =H\left(\varphi \circ h\left(e^{i \alpha_{0}} \xi, \xi\right), a\right) \leq \frac{1}{2 \pi} \int_{0}^{2 \pi} H\left(\varphi \circ h\left(\xi, e^{i \theta}\right), a\right) d \theta \\
& \leq \frac{1}{2 \pi} \int_{S} H\left(\varphi \circ h\left(\xi, e^{i \theta}\right), a\right) d \theta<\frac{1}{2 \pi} \int_{S} \log \left|\sigma\left(e^{i \theta}\right)\right| d \theta+\varepsilon \\
& =\frac{1}{2 \pi} \int_{0}^{2 \pi} \log \left|\sigma\left(e^{i \theta}\right)\right| d \theta+\varepsilon-\frac{1}{2 \pi} \int_{[0,2 \pi) \backslash S} \log \left|\sigma\left(e^{i \theta}\right)\right| d \theta \\
& <A+3 \varepsilon+C \varepsilon-\frac{\varepsilon}{\pi} \log \frac{\delta}{2} .
\end{aligned}
$$

Hence, $g_{D}^{2}(z)<A+3 \varepsilon+C \varepsilon-(\varepsilon / \pi) \log (\delta / 2)$. Since $\varepsilon>0$ was arbitrary the proof is complete.

LEMMA 10. $g_{D}(a, z)=g_{D}^{5}(a, z)$. 
Before we go into the proof of Lemma 10 recall the following result (see [P2]):

Theorem 11 (Poletsky). Let $G$ be a domain in $\mathbb{C}^{n}$ and let $u$ be an upper semicontinuous function in $G$. Then

$$
\widetilde{u}(w)=\inf \left\{\frac{1}{2 \pi} \int_{0}^{2 \pi} u\left(\phi\left(e^{i \theta}\right)\right) d \theta: \phi \in \mathcal{O}(\bar{E}, G), \phi(0)=w\right\}, \quad w \in G,
$$

is a plurisubharmonic function in $G$. Moreover, it is equal to the supremum of all plurisubharmonic functions $v$ such that $v \leq u$.

Proof of Lemma 10. Let us show first that for any $a \in D$ the function $k_{D}(a, \cdot)$ is upper semicontinuous in $D$.

Let $z_{0} \neq a$ and $k_{D}\left(a, z_{0}\right)<A$. There exists a holomorphic mapping $\varphi: \bar{E} \rightarrow D$ such that $\varphi(0)=z_{0}, \varphi(\sigma)=a, \sigma>0$, and $\log \sigma<A$. Let

$$
\varphi_{w}(\lambda):=\varphi(\lambda)+\left(w-z_{0}\right)(1-\lambda / \sigma), \quad \lambda \in \bar{E} .
$$

For some neighborhood $V$ of $z_{0}$ we have $\varphi_{w}(\bar{E}) \subset D, w \in V$. Note that $\varphi_{w}(0)=w$ and $\varphi_{w}(\sigma)=a$. Hence,

$$
k_{D}(a, w)<A, \quad w \in V .
$$

Assume now that $z_{0}=a$. Then $k_{D}\left(a, z_{0}\right)=-\infty$. Fix $A<0$ and let $\varphi_{w}(\lambda):=w+\lambda e^{-A}(a-w)$. Note that $\varphi_{w}(0)=w$ and $\varphi_{w}\left(e^{A}\right)=a$. For some neighborhood $V$ of $a$ we have $\varphi_{w}(\bar{E}) \subset D, w \in V$. Hence, $k_{D}(a, w) \leq$ $\log e^{A}=A, w \in V$.

Hence, by Theorem 11, we conclude that $g_{D}^{5}$ is a plurisubharmonic function which is a supremum over all plurisubharmonic functions not greater than $k_{D}$. But so is $g_{D}$, because $g_{D}(a, w) \leq k_{D}(a, w) \leq \log \|w-a\|-\log R$, $w \in B(a, R)$, where $R$ is such that $B(a, R) \subset D$.

LEMma 12. $g_{D}(a, z) \leq g_{D}^{4}(a, z)$.

Proof. Let $u \in \operatorname{PSH}(D), u<0$, be such that for some $M>0$ we have

$$
u(w) \leq M+\log \|w-a\| \quad \text { for } w \text { near } a .
$$

Take $\varphi \in \mathcal{O}(\bar{E}, D)$ with $\varphi(0)=z$ and $a \in \varphi(E)$. Let $\lambda_{j}, j=1, \ldots, N$, denote the solutions in $E$ of the equation $\varphi(\lambda)=a$ without multiplicity (if one takes solutions with multiplicities then one will get the inequality $g_{D}(a, z) \leq g_{D}^{2}(a, z)$, cf. [J-P], Chapter 4). Define

$$
f(\lambda):=\prod_{j=1}^{N} \frac{\lambda-\lambda_{j}}{1-\bar{\lambda}_{j} \lambda}
$$

Put $v:=u \circ \varphi-\log |f|$. It is clear that $v$ is a subharmonic function in $E \backslash\left\{\lambda_{1}, \ldots, \lambda_{N}\right\}$ and $v$ is locally bounded above on $E$. Hence $v$ extends 
subharmonically to $E$. By the maximum principle $v \leq 0$. In particular,

$$
u(z)=u(\varphi(0)) \leq \log |f(0)|=\sum_{j=1}^{N} \log \left|\lambda_{j}\right| .
$$

Hence $g_{D}(a, z) \leq g_{D}^{4}(a, z)$.

Acknowledgements. The ideas of the paper came after fruitful discussions with Professors M. Jarnicki and W. Zwonek. I would like to thank them. I would also like to thank the referee whose suggestions and corrections improved this paper.

\section{References}

[H] K. Hoffman, Banach Spaces of Analytic Functions, Prentice-Hall, 1962.

[J-P] M. Jarnicki and P. Pflug, Invariant Distances and Metrics in Complex Analysis, de Gruyter, 1993.

[K] M. Klimek, Pluripotential Theory, Oxford Univ. Press, 1991.

[N] K. Noshiro, Cluster Sets, Springer, 1960.

[P1] E. A. Poletsky, Holomorphic currents, Indiana Univ. Math. J. 42 (1993), 85-144.

[P2] -, Plurisubharmonic functions as solutions of variational problems, in: Proc. Sympos. Pure Math. 52, Amer. Math. Soc., 1991, 163-171.

[P-S] E. A. Poletsky and B. V. Shabat, Invariant metrics, in: Several Complex Variables III, G. M. Khenkin (ed.), Springer, 1989, 63-112.

[R] W. Rudin, Real and Complex Analysis, McGraw-Hill, 1974.

Institute of Mathematics

Jagiellonian University

Reymonta 4

30-059 Kraków, Poland

E-mail: edigaria@im.uj.edu.pl 